Bundesgesundheitsbl 2017 - 60:151-162 DOI 10.1007/s00103-016-2493-6 Online publiziert:21. Dezember 2016 (c) Springer-Verlag Berlin Heidelberg 2016

CrossMark

\author{
Claudia Diederichs ${ }^{1,2} \cdot$ Hannelore Neuhauser ${ }^{1,2} \cdot$ Lars Kroll' $\cdot$ Cornelia Lange $^{1} \cdot$ \\ Gert Mensink ${ }^{1}$. Christina Dornquast ${ }^{3}$. Christin Heidemann ${ }^{1}$. Christa Scheidt- \\ Nave ${ }^{1} \cdot$ Markus Busch ${ }^{1}$ \\ 'Abteilung für Epidemiologie und Gesundheitsmonitoring, Robert Koch-Institut, Berlin, Deutschland \\ ${ }_{2}^{2}$ partner site Berlin, DZHK (German Center for Cardiovascular Research), Berlin, Deutschland \\ ${ }^{3}$ Institut für Sozialmedizin, Epidemiologie und Gesundheitsökonomie, Charité - Universitätsmedizin \\ Berlin, Berlin, Deutschland
}

\section{Regionale Unterschiede in der Prävalenz von kardiovaskulären Risikofaktoren bei Männern und Frauen in Deutschland}

[10]. Regionale Unterschiede in der Prävalenz und Mortalität von HerzKreislauf-Erkrankungen wurden daher schon seit den 1980er-Jahren im Kontext einer unterschiedlichen Verteilung von kardiovaskulären Risikofaktoren in der Allgemeinbevölkerung diskutiert [3].

Für Deutschland wurden kürzlich erstmals Daten aus der Todesursachenstatistik, verschiedenen epidemiologischen Studien und Erhebungen der Sozialberichterstattung zusammengestellt und bemerkenswerte Parallelen zwischen Bundeslandunterschieden in der Mortalität der ischämischen Herzkrankheit und der Prävalenz von individuellen Risikofaktoren und sozialen Indikatoren, wie zum Beispiel Arbeitslosigkeit, gezeigt [11]. Bisher gibt es dazu aber keine Ergebnisse auf Basis von Individualdaten, die auch Rückschlüsse auf die regionale Kumulation von verschiedenen kardiovaskulären Risikofaktoren oder eine Differenzierung nach Geschlecht zulassen.

Vor diesem Hintergrund erfolgt nun eine Analyse der bevölkerungsbezogenen Studie „Gesundheit in Deutschland aktuell (GEDA)“ 2010-2012 mit Daten $\mathrm{zu}$ acht klassischen kardiovaskulären Risikofaktoren sowie zur sozialen Lage der Teilnehmer, wobei bei über 62.000 Teilnehmern Bundeslandunterschiede auch erstmals geschlechtsspezifisch untersucht werden können.

\section{Methode}

\section{Studiendesign und Stichprobe}

Die nachfolgenden Analysen beruhen auf gepoolten Daten der Erhebungswellen 2009, 2010 und 2012 des bundesweit repräsentativen Gesundheitssurveys „Gesundheit in Deutschland aktuell (GEDA)“, in denen insgesamt 62.606 Personen über 18 Jahren in Privathaushalten mithilfe eines computergestützten telefonischen Interviews befragt wurden [12]. Die Daten der drei unabhängigen Wellen wurden zusammengefasst, um die Teststärke speziell für die Untersuchung von Unterschieden zwischen Untergruppen, in diesem Fall den 16 Ländern, zu erhöhen [8]. Der Anteil der realisierten Interviews an allen wahrscheinlichen Haushalten betrug 34,5\% (GEDA 2009), $35,2 \%$ (GEDA 2010) und 23,9\% (GEDA 2012). Die Kooperationsrate der erreichten Zielpersonen betrug 51,2\% (GEDA 2009), 55,8 \% (GEDA 2010) und $76,6 \%$ (GEDA 2012). Detaillierte Informationen zum Studiendesign und zur Stichprobe finden sich hier [12-15].

\section{Kardiovaskuläre Risikofaktoren}

Hinsichtlich der verhaltensbezogenen Risikofaktoren wurden Teilnehmer, die nach eigenen Angaben in den letzten drei Monaten keinen Sport getrieben 


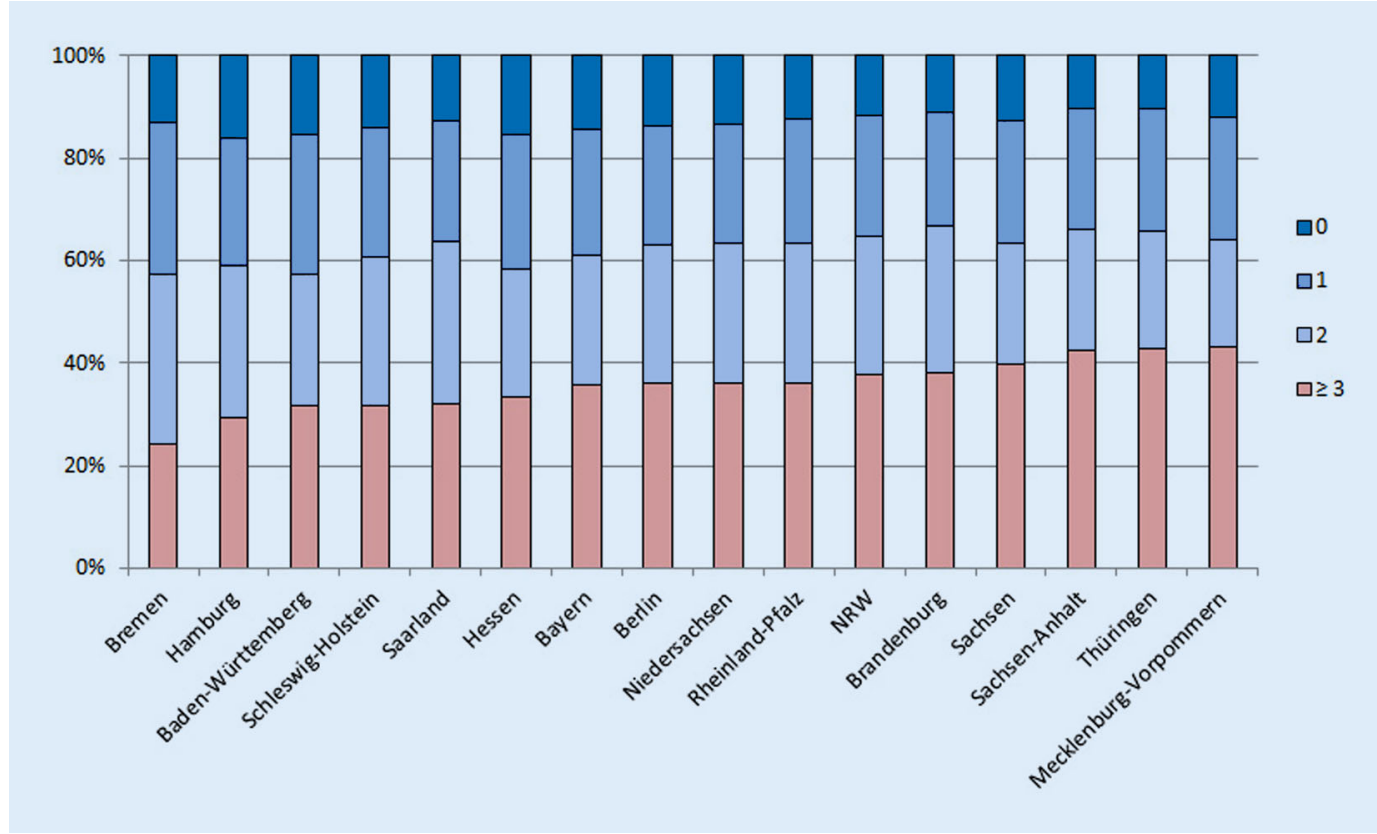

Abb. $1 \varangle$ Verteilung der Anzahl der Risikofaktoren bei Männern nach Bundesland $(n=26.992)$

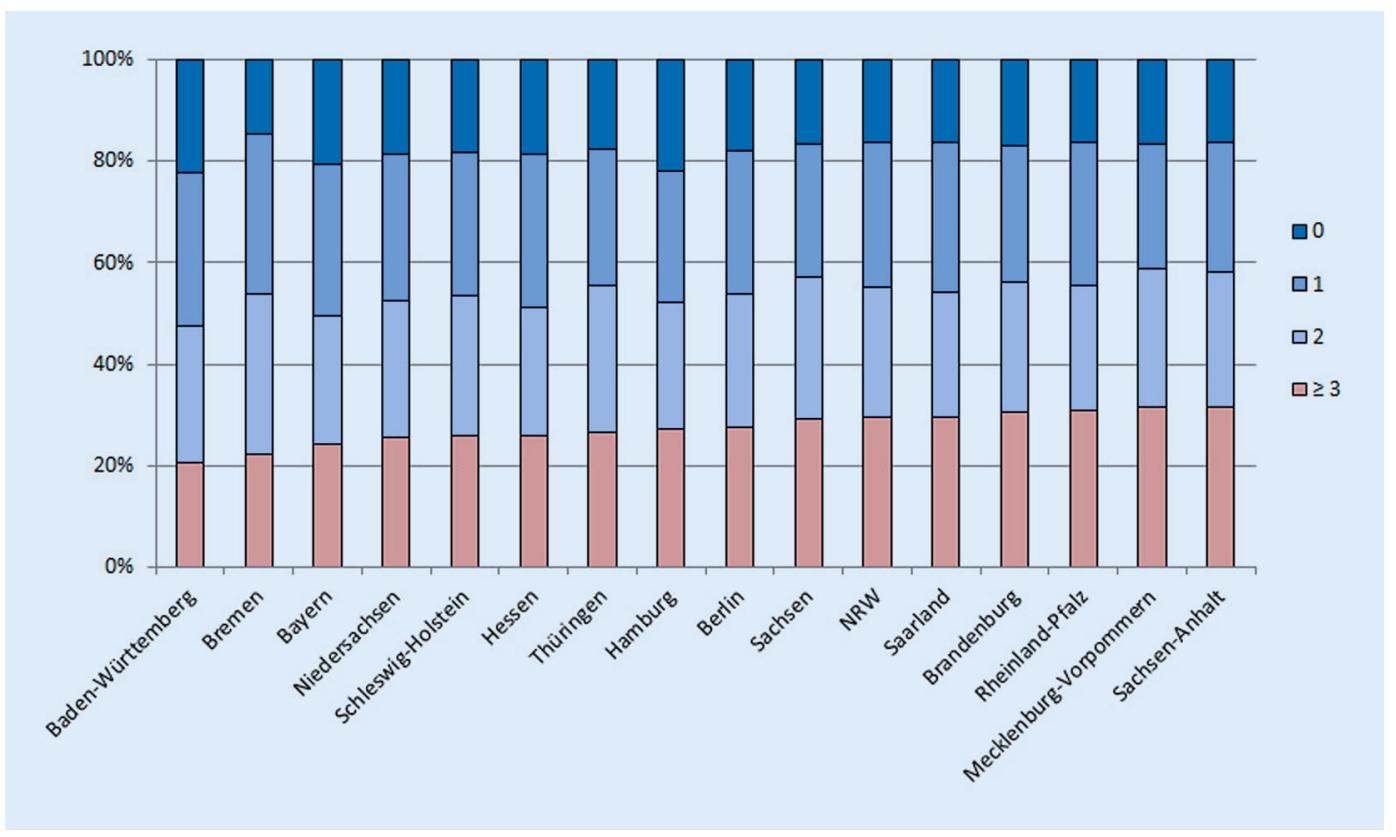

Abb. $2 \triangleleft$ Verteilung der Anzahl der Risikofaktoren bei Frauen nach Bundesland $(n=32.739)$

hatten, als sportlich inaktiv bezeichnet. Ein riskanter Alkoholkonsum wurde mit dem „Alcohol Use Disorder Identification Test - Consumption (AUDITC)“ erfasst und als ein Punktwert $\geq 4$ bei Frauen und $\geq 5$ von 12 bei Männern definiert [16]. Teilnehmer wurden als Raucher klassifiziert, wenn sie angaben, aktuell entweder täglich oder gelegentlich zu rauchen. Der tägliche Obst- und Gemüseverzehr wurde als gering bezeichnet, falls die Teilnehmer weniger als eine Portion Obst, Gemü- se oder Saft am Tag zu sich nahmen. Bei den krankheitsnahen Risikofaktoren wurde der Body-Mass-Index (BMI) auf Grundlage von Angaben zur Größe und zum Gewicht berechnet. Adipositas wurde als $\mathrm{BMI} \geq 30 \mathrm{~kg} / \mathrm{m}^{2}$ definiert. Die Lebenszeitprävalenzen von Hypertonie, Diabetes und Fettstoffwechselstörungen beruhen auf Selbstangaben zu jemals ärztlich diagnostizierten Erkrankungen. Antworten mit „Weiß nicht“ oder „Keine Angabe“ wurden für alle Risikofaktoren als fehlender Wert klassifiziert.

\section{Weitere Variablen}

Daten zu Alter, Geschlecht und Bundesland beruhten auf Selbstangaben. Der Sozialstatus wurde mit einem Index basierend auf Informationen zu schulischer und beruflicher Bildung, beruflicher Stellung und Nettoäquivalenzeinkommen erfasst und verteilungsbasiert als niedrig, mittel oder hoch klassifiziert [17]. In den logistischen Regressionsmodellen wurden fehlende Angaben zum Sozialstatus $(n=89,0,2 \%)$ als eine eigene Vari- 
Bundesgesundheitsbl 2017·60:151-162 DOI 10.1007/s00103-016-2493-6

(c) Springer-Verlag Berlin Heidelberg 2016

\section{Diederichs $\cdot$ H. Neuhauser $\cdot$ L. Kroll · C. Lange · G. Mensink · C. Dornquast · C. Heidemann · C. Scheidt-Nave $\cdot$ M. Busch}

\section{Regionale Unterschiede in der Prävalenz von kardiovaskulären Risikofaktoren bei Männern und Frauen in Deutschland}

\section{Zusammenfassung}

Hintergrund. Über die Hälfte aller kardiovaskulären Erkrankungen wird von acht zum Teil vermeidbaren Risikofaktoren verursacht.

Ziele. Angesichts deutlicher Prävalenz- und Mortalitätsunterschiede bei Herz-KreislaufErkrankungen zwischen den deutschen Bundesländern wird die regionale Verteilung von kardiovaskulären Risikofaktoren bevölkerungsrepräsentativ und geschlechtsspezifisch untersucht.

Methoden. Anhand von gepoolten Daten ( $n=$ 62.606) der bundesweiten, telefonischen $\mathrm{Ge}$ sundheitssurveys "Gesundheit in Deutschland aktuell (GEDA)" 2009, 2010 und 2012 wurden die Prävalenz von sportlicher Inaktivität, riskantem Alkoholkonsum, Rauchen, geringem Obst- und Gemüseverzehr, Adipositas, ärztlich diagnostizierter Hypertonie, Diabetes und Fettstoffwechselstörungen sowie die zusammengefasste Anzahl von Risikofaktoren getrennt für Männer und Frauen in den Bundesländern bestimmt. Zusätzlich wurde der Einfluss von Alter und Sozialstatus auf Prävalenzunterschiede untersucht. Ergebnisse. Im Bundesdurchschnitt hatten $36,0 \%$ der männlichen und $26,6 \%$ der weiblichen Bevölkerung drei oder mehr Risikofaktoren. Große Unterschiede zwischen Männern und Frauen gab es vor allem beim riskanten Alkoholkonsum (32,8 \% versus $21,7 \%$ ), einem geringen Obst- und Gemüseverzehr (20,6 \% versus 10,4\%) sowie beim aktuellen Rauchen (32,6\% versus $24,9 \%$ ). Bei allen acht Risikofaktoren wurden deutliche Prävalenzunterschiede zwischen den Bundesländern gefunden. In den ostdeutschen Bundesländern mit Ausnahme von Berlin wurden die höchsten Prävalenzen von sportlicher Inaktivität, Adipositas, Hypertonie und Diabetes bei beiden Geschlechtern und von riskantem Alkoholkonsum bei Männern beobachtet. Sachsen-Anhalt war das einzige Bundesland mit den höchsten Prävalenzen für zwei Risikofaktoren. Beim Rauchen nahmen die drei Stadtstaaten Berlin,
Hamburg und Bremen vordere Rangplätze ein. Der Anteil der Bevölkerung mit weniger als einer Portion Obst, Gemüse oder Saft pro Tag war geschlechtsübergreifend im Saarland am höchsten. Die regionalen Unterschiede blieben auch nach Adjustierung für Alter und sozialen Status bestehen.

Diskussion. Die Verteilung der Risikofaktoren zwischen den Bundesländern zeigt ähnliche regionale Unterschiede wie die Prävalenz und Mortalität von Herz-Kreislauf-Erkrankungen mit einer ungünstigeren Situation in den ostdeutschen Ländern mit Ausnahme von Berlin. Insgesamt ist für ganz Deutschland ein erheblicher Bedarf hinsichtlich der Prävention von größtenteils modifizierbaren Risikofaktoren für Herz-Kreislauf-Erkrankungen bei Männern und Frauen erkennbar.

Schlüsselwörter

Risikofaktoren · Regionale Unterschiede . Kardiovaskuläre Erkrankungen · Hypertonie · Diabetes

\section{Regional differences in the prevalence of cardiovascular risk factors in men and women in Germany}

\section{Abstract}

Background. More than half of all cardiovascular diseases are caused by eight, mostly preventable risk factors.

Objectives. In view of the considerable differences in the prevalence and mortality of cardiovascular diseases between the 16 German federal states, the regional distribution of cardiovascular risk factors was analyzed stratified for men and women, using population-based data.

Methods. Pooled data $(n=62,606)$ from the national, telephone health surveys "German Health Update" from 2009, 2010 and 2012 were used to estimate the prevalence of physical inactivity, risky alcohol consumption, smoking, low fruit and vegetable consumption, obesity and diagnosed hypertension, diabetes and dyslipidemia and the accumulated number of risk factors stratified for men and women in the federal states. Furthermore, we analyzed the influence of age and social status on prevalence differences.

Results. At the national level, $36.0 \%$ of men and $26.6 \%$ of women had three or more risk factors. Large differences between men and women were found for risky alcohol consumption (32.8\% versus $21.7 \%$ ), low fruit and vegetable consumption $(20.6 \%$ versus $10.4 \%$ ) and current smoking (32.6\% versus $24.9 \%)$. The prevalence of all eight risk factors differed considerably between federal states. The highest prevalence of physical inactivity, obesity, hypertension and diabetes in both sexes as well as risky alcohol consumption in men were observed in the Eastern federal states (except for Berlin). Sachsen-Anhalt was the only federal state with the highest prevalence for two risk factors. Current smoking was most prevalent in the three federal city states Berlin, Hamburg and
Bremen. Saarland had the highest prevalence of low fruit and vegetable consumption in both sexes. Regional differences remained after adjustment for age and social status. Conclusions. There is evidence for regional differences in cardiovascular risk factor levels in Germany that resemble variations in the prevalence and mortality of cardiovascular diseases between federal states with a more unfavorable situation in the East (except for Berlin). Overall, this study shows a considerable need for the prevention of mostly modifiable risk factors for cardiovascular diseases in men and women in Germany.

\section{Keywords}

Risk factors - Regional differences - Cardiovascular diseases . Hypertension . Diabetes 
Tab. 1 Beschreibung der Teilnehmer der GEDA-Studie 2009-2012 (in Prozent, gewichtet ${ }^{a}$ )

\begin{tabular}{|c|c|c|c|}
\hline & & $\begin{array}{l}\text { Männer } \\
(n=28.033)\end{array}$ & $\begin{array}{l}\text { Frauen } \\
(n=34.573)\end{array}$ \\
\hline \multirow[t]{6}{*}{ Alter } & 18-34 Jahre & 25,4 & 23,1 \\
\hline & 35-44 Jahre & 17,0 & 15,6 \\
\hline & 45-54 Jahre & 20,7 & 19,0 \\
\hline & 55-64 Jahre & 15,5 & 15,0 \\
\hline & 65-74 Jahre & 14,0 & 15,3 \\
\hline & $\geq 75$ Jahre & 7,5 & 12,1 \\
\hline \multirow{3}{*}{$\begin{array}{l}\text { Sozioökonomischer } \\
\text { Status }\end{array}$} & Niedrig & 17,2 & 21,0 \\
\hline & Mittel & 58,6 & 60,7 \\
\hline & Hoch & 24,2 & 18,4 \\
\hline \multirow[t]{16}{*}{ Wohnregion } & Schleswig-Holstein & 3,4 & 3,5 \\
\hline & Hamburg & 2,2 & 2,2 \\
\hline & Niedersachsen & 9,6 & 9,5 \\
\hline & Bremen & 0,8 & 0,8 \\
\hline & NRW & 21,5 & 21,8 \\
\hline & Hessen & 7,4 & 7,4 \\
\hline & Rheinland-Pfalz & 4,9 & 4,9 \\
\hline & Baden-Württemberg & 13,1 & 13,0 \\
\hline & Bayern & 15,3 & 15,3 \\
\hline & Saarland & 1,2 & 1,3 \\
\hline & Berlin & 4,4 & 4,4 \\
\hline & Brandenburg & 3,2 & 3,1 \\
\hline & Mecklenburg-Vorpommern & 2,1 & 2,0 \\
\hline & Sachsen & 5,2 & 5,2 \\
\hline & Sachsen-Anhalt & 2,9 & 2,9 \\
\hline & Thüringen & 2,8 & 2,8 \\
\hline
\end{tabular}

able kodiert, um Verzerrungen durch fehlende Angaben zu vermeiden.

\section{Statistische Analyse}

Die Daten aus den drei GEDA-Wellen 2009, 2010 und 2012 wurden gepoolt und anschließend mithilfe eines Gewichtungsfaktors an die Alters-, Geschlechts-, Bildungs- und Regionalverteilung der Bevölkerung in Deutschland zum 31. Dezember 2011 angepasst, um mögliche Unterschiede im Response-Verhalten auszugleichen [12-15]. Für jedes Bundesland wurde die rohe Prävalenz aller acht kardiovaskulären Risikofaktoren mit Angabe des 95\%-Konfidenzintervalls stratifiziert für Männer und Frauen berechnet. Für jeden Risikofaktor wurden Teilnehmer mit fehlenden Werten (maximal 1,2\% pro Risikofaktor) von der Analyse ausgeschlossen. Um Unter- schiede zwischen den einzelnen Ländern für Alter und den soziodemografischen Status zu kontrollieren, wurde für jeden Risikofaktor ein logistisches Regressionsmodell erstellt und das Bundesland mit der niedrigsten Prävalenz jeweils als Referenzkategorie festgelegt. Im letzten Schritt wurden der Anteil der Bevölkerung mit null, einem, zwei und drei oder mehr Risikofaktoren getrennt für Frauen und Männer berechnet und die Bundesländer in aufsteigender Reihenfolge nach der Prävalenz von drei oder mehr Risikofaktoren angeordnet. Für alle Auswertungen wurden Surveyprozeduren des Statistikprogramms Stata SE14 (StataCorp LP, Texas, US) verwendet.

\section{Ergebnisse}

Insgesamt nahmen 62.606 Menschen an den drei GEDA-Befragungen teil, davon waren 48,9 \% Männer. Das mittlere Alter betrug 48,4 Jahre (Spannweite: 18-99) in der männlichen und 50,9 Jahre (Spannweite: 18-100) in der weiblichen Studienpopulation (• Tab. 1).

Bei beiden Geschlechtern (• Tab. 2 und 3) gab es deutliche Unterschiede zwischen den Ländern bei allen acht Risikofaktoren. Diabetes hatte die niedrigste Prävalenz mit einer regionalen Spannweite zwischen 3,6 und $13,4 \%$ in der männlichen sowie 7,2 und 13,6\% in der weiblichen Bevölkerung. Sportliche Inaktivität war von allen Risikofaktoren am häufigsten und lag je nach Bundesland bei den Männern zwischen 31,7 und $44,1 \%$ und bei den Frauen zwischen 31,0 und $41,2 \%$.

Auch wenn kein einzelnes Land für alle Risikofaktoren die höchsten Prävalenzen aufwies, zeigten sich insbesondere bei den Männern deutliche Unterschiede zwischen den west- und ostdeutschen Bundesländern. Bei den Männern lagen bei fünf, beziehungsweise bei den Frauen bei vier von acht Risikofaktoren die höchsten Prävalenzen im Osten mit Ausnahme von Berlin. Dies galt für die Risikofaktoren sportliche Inaktivität, Adipositas, Hypertonie und Diabetes bei beiden Geschlechtern und zusätzlich noch für riskanten Alkoholkonsum bei den Männern. Sachsen-Anhalt war das einzige Bundesland mit den höchsten Prävalenzen für zwei Risikofaktoren sowohl bei Männern als auch bei Frauen. Fettstoffwechselstörungen hingegen wurden häufiger im Westen beobachtet. Die regionalen Unterschiede blieben auch nach Adjustierung für Alter und sozialem Status bestehen (-Tab. 4 und 5).

Zudem waren weitere regionale Muster erkennbar: Der Anteil der Bevölkerung mit weniger als einer Portion Obst, Gemüse oder Saft pro Tag war geschlechtsübergreifend im Saarland am höchsten und in Sachsen am niedrigsten. Bei der Prävalenz des Rauchens nahmen die drei Stadtstaaten Berlin, Hamburg und Bremen bei Männern und Frauen vordere Rangplätze ein, während in Sachsen bei beiden Geschlechtern der bundesweit niedrigste Anteil von Rauchern gefunden wurde. Auf der anderen Seite waren die Prävalenzen vieler anderer Risikofaktoren in Bremen und 
Tab. 2 Prävalenz kardiovaskulärer Risikofaktoren bei Männern nach Bundesland (\%, 95\%-Konfidenzintervall [KI], gewichtet). (Alle Prozentangaben

sind durch Gewichtung an die Bevölkerung in Deutschland am 31.12.2011 angepasst. In absteigender Reihenfolge sind die zwei höchsten Prävalenzen jeweils rot und hellrot und die niedrigsten Prävalenz hellgrün und grün markiert)

\begin{tabular}{|c|c|c|c|c|c|c|c|c|}
\hline & $\begin{array}{c}\text { Keine } \\
\text { sportliche } \\
\text { Aktivität } \\
(n=28.013)\end{array}$ & $\begin{array}{c}\text { Riskanter } \\
\text { Alkohol- } \\
\text { konsum } \\
(n=27.789)\end{array}$ & $\begin{array}{c}\text { Aktuelles } \\
\text { Rauchen } \\
(n=28.024)\end{array}$ & $\begin{array}{c}<1 \text { Portion } \\
\text { Obst/ } \\
\text { Gemüse/ } \\
\text { Saft pro } \\
\text { Tag } \\
(n=27.698)\end{array}$ & $\begin{array}{c}\text { Adipositas } \\
(\mathrm{BMI} \geq 30) \\
(n=27.768)\end{array}$ & $\begin{array}{c}\text { Hypertonie } \\
\text { (jemals } \\
\text { Diagnose) } \\
(n=27.971)\end{array}$ & $\begin{array}{l}\text { Diabetes } \\
\text { (jemals } \\
\text { Diagnose) } \\
(n=28.011)\end{array}$ & $\begin{array}{c}\text { Fettstoff } \\
\text { wechsel- } \\
\text { Störung } \\
\text { (jemals } \\
\text { Diagnose) } \\
\text { ( } n=27.856)\end{array}$ \\
\hline $\begin{array}{l}\text { Schleswig- } \\
\text { Holstein }\end{array}$ & $\begin{array}{c}34,0 \\
30,2-38,0\end{array}$ & $\begin{array}{c}27,4 \\
24,0-31,1\end{array}$ & $\begin{array}{c}33,4 \\
29,6-37,3\end{array}$ & $\begin{array}{c}18,7 \\
15,6-22,0\end{array}$ & $\begin{array}{c}16,1 \\
13,2-19,5\end{array}$ & $\begin{array}{c}33,4 \\
29,6-37,3\end{array}$ & $\begin{array}{c}9,8 \\
7,4-12,7\end{array}$ & $\begin{array}{c}27,1 \\
23,6-30,8\end{array}$ \\
\hline Hamburg & $\begin{array}{c}34,8 \\
29,7-40,3\end{array}$ & $\begin{array}{c}30,4 \\
26,0-35,2\end{array}$ & $\begin{array}{c}36,9 \\
32,0-42,1\end{array}$ & $\begin{array}{c}18,3 \\
14,6-22,7\end{array}$ & $\begin{array}{c}10,4 \\
7,7-13,8\end{array}$ & $\begin{array}{c}23,9 \\
19,8-25,6\end{array}$ & $\begin{array}{c}5,8 \\
3,7-9,0\end{array}$ & $\begin{array}{c}32,5 \\
27,7-37,6\end{array}$ \\
\hline $\begin{array}{l}\text { Nieder- } \\
\text { sachsen }\end{array}$ & $\begin{array}{c}36,4 \\
34,1-38,7\end{array}$ & $\begin{array}{c}33,2 \\
31,0-35,4\end{array}$ & $\begin{array}{c}31,8 \\
29,6-34,0\end{array}$ & $\begin{array}{c}19,1 \\
17,3-21,6\end{array}$ & $\begin{array}{c}17,5 \\
15,7-19,4\end{array}$ & $\begin{array}{c}33,8 \\
31,6-36,2\end{array}$ & $\begin{array}{c}7,0 \\
5,9-8,3\end{array}$ & $\begin{array}{c}29,1 \\
26,9-31,3\end{array}$ \\
\hline Bremen & $\begin{array}{c}38,9 \\
30,6-48,0\end{array}$ & $\begin{array}{c}26,0 \\
19,6-33,5\end{array}$ & $\begin{array}{c}34,2 \\
26,4-43,0\end{array}$ & $\begin{array}{c}16,2 \\
10,8-23,6\end{array}$ & $\begin{array}{c}13,2 \\
7,8-21,4\end{array}$ & $\begin{array}{c}29,0 \\
21,5-37,7\end{array}$ & $\begin{array}{c}3,6 \\
1,6-7,7\end{array}$ & $\begin{array}{c}28,4 \\
21,1-37,0\end{array}$ \\
\hline NRW & $\begin{array}{c}36,0 \\
34,3-37,7\end{array}$ & $\begin{array}{c}34,2 \\
32,7-35,8\end{array}$ & $\begin{array}{c}35,3 \\
33,6-36,9\end{array}$ & $\begin{array}{c}20,2 \\
18,9-21,7\end{array}$ & $\begin{array}{c}16,9 \\
15,5-18,3\end{array}$ & $\begin{array}{c}32,2 \\
30,5-33,8\end{array}$ & $\begin{array}{c}8,6 \\
7,6-9,8\end{array}$ & $\begin{array}{c}33,1 \\
31,4-34,8\end{array}$ \\
\hline Hessen & $\begin{array}{c}36,0 \\
33,5-38,6\end{array}$ & $\begin{array}{c}30,6 \\
28,3-33,0\end{array}$ & $\begin{array}{c}32,3 \\
29,9-34,8\end{array}$ & $\begin{array}{c}19,4 \\
17,4-21,6\end{array}$ & $\begin{array}{c}15,7 \\
13,9-17,8\end{array}$ & $\begin{array}{c}30,4 \\
28,0-32,9\end{array}$ & $\begin{array}{c}8,6 \\
7,2-9,8\end{array}$ & $\begin{array}{c}28,4 \\
26,1-30,8\end{array}$ \\
\hline $\begin{array}{l}\text { Rheinland- } \\
\text { Pfalz }\end{array}$ & $\begin{array}{c}32,2 \\
29,1-35,5\end{array}$ & $\begin{array}{c}33,1 \\
30,1-36,2\end{array}$ & $\begin{array}{c}30,3 \\
27,3-33,4\end{array}$ & $\begin{array}{c}22,2 \\
19,5-25,2\end{array}$ & $\begin{array}{c}17,6 \\
15,1-20,3\end{array}$ & $\begin{array}{c}33,1 \\
30,0-36,4\end{array}$ & $\begin{array}{c}9,1 \\
7,2-11,5\end{array}$ & $\begin{array}{c}34,7 \\
31,6-38,0\end{array}$ \\
\hline $\begin{array}{l}\text { Baden - } \\
\text { Württemberg }\end{array}$ & $\begin{array}{c}31,7 \\
29,6-33,7\end{array}$ & $\begin{array}{c}28,6 \\
26,8-30,5\end{array}$ & $\begin{array}{c}29,9 \\
28,0-31,9\end{array}$ & $\begin{array}{c}21,6 \\
19,9-23,5\end{array}$ & $\begin{array}{c}14,2 \\
12,7-15,8\end{array}$ & $\begin{array}{c}28,1 \\
26,2-30,1\end{array}$ & $\begin{array}{c}7,4 \\
6,3-8,7\end{array}$ & $\begin{array}{c}30,8 \\
28,8-32,8\end{array}$ \\
\hline Bayern & $\begin{array}{c}33,3 \\
31,4-32,3\end{array}$ & $\begin{array}{c}32,9 \\
31,1-34,7\end{array}$ & $\begin{array}{c}30,6 \\
28,8-32,3\end{array}$ & $\begin{array}{c}23,9 \\
22,2-25,6\end{array}$ & $\begin{array}{c}16,4 \\
14,9-17,8\end{array}$ & $\begin{array}{c}32,0 \\
30,2-33,9\end{array}$ & $\begin{array}{c}8,0 \\
7,0-9,2\end{array}$ & $\begin{array}{c}32,5 \\
30,7--34,3\end{array}$ \\
\hline Saarland & $\begin{array}{c}34,3 \\
28,0-41,3\end{array}$ & $\begin{array}{c}31,5 \\
25,8-37,9\end{array}$ & $\begin{array}{c}28,8 \\
23,1-35,2\end{array}$ & $\begin{array}{c}28,1 \\
22,6-34,4\end{array}$ & $\begin{array}{c}15,6 \\
11,3-21,0\end{array}$ & $\begin{array}{c}31,6 \\
25,5-38,3\end{array}$ & $\begin{array}{c}8,6 \\
5,5-13,2\end{array}$ & $\begin{array}{c}28,7 \\
23,3-34,8\end{array}$ \\
\hline Berlin & $\begin{array}{c}36,0 \\
32,7-39,5\end{array}$ & $\begin{array}{c}31,7 \\
28,6-34,9\end{array}$ & $\begin{array}{c}40,7 \\
37,3-44,2\end{array}$ & $\begin{array}{c}24,2 \\
21,3-27,5\end{array}$ & $\begin{array}{c}14,1 \\
11,7-16,9\end{array}$ & $\begin{array}{c}28,7 \\
25,7-31,8\end{array}$ & $\begin{array}{c}8,1 \\
6,4-10,3\end{array}$ & $\begin{array}{c}30,1 \\
27,0-33,3\end{array}$ \\
\hline Brandenburg & $\begin{array}{c}36,4 \\
33,0-40,1\end{array}$ & $\begin{array}{c}33,5 \\
30,2-36,9\end{array}$ & $\begin{array}{c}33,9 \\
30,6--37,4\end{array}$ & $\begin{array}{c}18,6 \\
16,0-21,6\end{array}$ & $\begin{array}{c}17,8 \\
15,2-20,7\end{array}$ & $\begin{array}{c}38,6 \\
35,2-31,1\end{array}$ & $\begin{array}{c}13,4 \\
10,9-16,4\end{array}$ & $\begin{array}{c}31,1 \\
28,0-34,5\end{array}$ \\
\hline $\begin{array}{l}\text { Mecklenburg- } \\
\text { Vorpommern }\end{array}$ & $\begin{array}{c}41,4 \\
36,4-46,6\end{array}$ & $\begin{array}{c}34,8 \\
29,9-40,0\end{array}$ & $\begin{array}{c}35,1 \\
30,3-40,2\end{array}$ & $\begin{array}{c}17,3 \\
13,6-21,8\end{array}$ & $\begin{array}{c}20,0 \\
16,1-24,6\end{array}$ & $\begin{array}{c}38,9 \\
34,0-44,0\end{array}$ & $\begin{array}{c}12,1 \\
9,2-15,7\end{array}$ & $\begin{array}{c}30,4 \\
25,9-35,3\end{array}$ \\
\hline Sachsen & $\begin{array}{c}38,6 \\
35,7-41,6\end{array}$ & $\begin{array}{c}40,1 \\
37,2-43,0\end{array}$ & $\begin{array}{c}28,3 \\
25,7-31,0\end{array}$ & $\begin{array}{c}15,4 \\
13,4-17,7\end{array}$ & $\begin{array}{c}17,3 \\
15,1-19,8\end{array}$ & $\begin{array}{c}37,5 \\
34,7-40,4\end{array}$ & $\begin{array}{c}12,7 \\
10,8-14,9\end{array}$ & $\begin{array}{c}28,3 \\
25,7--31,0\end{array}$ \\
\hline $\begin{array}{l}\text { Sachsen- } \\
\text { Anhalt }\end{array}$ & $\begin{array}{c}44,1 \\
39,8-48,5\end{array}$ & $\begin{array}{c}36,9 \\
32,8-41,3\end{array}$ & $\begin{array}{c}34,5 \\
30-38,8\end{array}$ & $\begin{array}{c}16,8 \\
13,6-20,4\end{array}$ & $\begin{array}{c}17,6 \\
14,5-21,2\end{array}$ & $\begin{array}{c}42,5 \\
38,2-46,9\end{array}$ & $\begin{array}{c}9,9 \\
7,5-12,9\end{array}$ & $\begin{array}{c}30,3 \\
26,3-34,5\end{array}$ \\
\hline Thüringen & $\begin{array}{c}35,4 \\
34,6-26,2\end{array}$ & $\begin{array}{c}39,1 \\
35,5-42,9\end{array}$ & $\begin{array}{c}30,9 \\
27,5-34,5\end{array}$ & $\begin{array}{c}20,6 \\
17,5-24,0\end{array}$ & $\begin{array}{c}18,4 \\
15,5-21,7\end{array}$ & $\begin{array}{c}40,7 \\
37,1-44,5\end{array}$ & $\begin{array}{c}11,8 \\
9,5-14,6\end{array}$ & $\begin{array}{c}29,3 \\
26,0-32,9\end{array}$ \\
\hline $\begin{array}{l}\text { Gesamt- } \\
\text { Deutschland }\end{array}$ & $\begin{array}{c}35,4 \\
34,6-36,2\end{array}$ & $\begin{array}{c}32,8 \\
32,2-33,5\end{array}$ & $\begin{array}{c}32,6 \\
31,9-33,3\end{array}$ & $\begin{array}{c}20,6 \\
20,0-21,2\end{array}$ & $\begin{array}{c}16,3 \\
15,7-16,9\end{array}$ & $\begin{array}{c}32,5 \\
31,8-33,2\end{array}$ & $\begin{array}{c}8,7 \\
8,3-9,2\end{array}$ & $\begin{array}{c}31,0 \\
30,3-31,7\end{array}$ \\
\hline
\end{tabular}

*alle Prozentangaben sind durch Gewichtung an die Bevölkerung in Deutschland am 31.12.2011 angepasst 
Tab. 3 Prävalenz kardiovaskulärer Risikofaktoren bei Frauen nach Bundesland (\%, 95\%-Kl, gewichtet). (Alle Prozentangaben sind durch Gewichtung an die Bevölkerung in Deutschland am 31.12.2011 angepasst. In absteigender Reihenfolge sind die zwei höchsten Prävalenzen jeweils rot und hellrot und die niedrigsten Prävalenz hellgrün und grün markiert)

\begin{tabular}{|c|c|c|c|c|c|c|c|c|}
\hline & $\begin{array}{c}\text { Keine } \\
\text { sportliche } \\
\text { Aktivität } \\
(n=34.540)\end{array}$ & $\begin{array}{c}\text { Riskanter } \\
\text { Alkohol- } \\
\text { konsum } \\
(n=34.312)\end{array}$ & $\begin{array}{c}\text { Aktuelles } \\
\text { Rauchen } \\
(n=34.563)\end{array}$ & $\begin{array}{c}<1 \text { Portion } \\
\text { Obst/ } \\
\text { Gemüise/ } \\
\text { Saft pro } \\
\text { Tag } \\
(n=34.152)\end{array}$ & $\begin{array}{c}\text { Adipositas } \\
(\mathrm{BMI} \geq 30) \\
(n=33.563)\end{array}$ & $\begin{array}{c}\text { Hypertonie } \\
\text { (jemals } \\
\text { Diagnose) } \\
(n=34.522)\end{array}$ & $\begin{array}{c}\text { Diabetes } \\
\text { (jemals } \\
\text { Diagnose) } \\
(n=34.543)\end{array}$ & $\begin{array}{c}\text { Fettstoff } \\
\text { wechsel- } \\
\text { störung } \\
\text { (jemals } \\
\text { Diagnose) } \\
(n=34.409)\end{array}$ \\
\hline $\begin{array}{l}\text { Schleswig- } \\
\text { Holstein }\end{array}$ & $\begin{array}{c}34,2 \\
30,8-37,8\end{array}$ & $\begin{array}{c}21,1 \\
18,4-24,0\end{array}$ & $\begin{array}{c}27,8 \\
24,1-31,1\end{array}$ & $\begin{array}{c}8,9 \\
7,0-11,3\end{array}$ & $\begin{array}{c}14,3 \\
11,7-17,4\end{array}$ & $\begin{array}{c}29,5 \\
26,3-33,0\end{array}$ & $\begin{array}{c}7,9 \\
6,0-10,4\end{array}$ & $\begin{array}{c}28,3 \\
25,0-31,9\end{array}$ \\
\hline Hamburg & $\begin{array}{c}33,4 \\
29,1-37,9\end{array}$ & $\begin{array}{c}24,6 \\
21,1-28,5\end{array}$ & $\begin{array}{c}30,6 \\
26,6-34,9\end{array}$ & $\begin{array}{c}8,8 \\
6,5-11,9\end{array}$ & $\begin{array}{c}14,2 \\
11,0-18,1\end{array}$ & $\begin{array}{c}30,4 \\
26,3-34,8\end{array}$ & $\begin{array}{c}8,4 \\
6,0-11,6\end{array}$ & $\begin{array}{c}26,2 \\
22,4-30,4\end{array}$ \\
\hline $\begin{array}{l}\text { Nieder- } \\
\text { sachsen }\end{array}$ & $\begin{array}{c}34,3 \\
32,1-36,1\end{array}$ & $\begin{array}{c}20,9 \\
19,3-22,6\end{array}$ & $\begin{array}{c}24,2 \\
22,5-26,1\end{array}$ & $\begin{array}{c}10,4 \\
9,1-11,9\end{array}$ & $\begin{array}{c}15,7 \\
14,0-17,5\end{array}$ & $\begin{array}{c}30,9 \\
28,7-33,2\end{array}$ & $\begin{array}{c}8,3 \\
7,0-9,9\end{array}$ & $\begin{array}{c}27,3 \\
25,2-29,4\end{array}$ \\
\hline Bremen & $\begin{array}{c}33,6 \\
26,9-41,0\end{array}$ & $\begin{array}{c}23,8 \\
18,5-30,0\end{array}$ & $\begin{array}{c}30,9 \\
24,6-38,0\end{array}$ & $\begin{array}{c}10,2 \\
6,3-15,9\end{array}$ & $\begin{array}{c}10,4 \\
6,5-16,2\end{array}$ & $\begin{array}{c}27,4 \\
21,3-34,4\end{array}$ & $\begin{array}{c}9,6 \\
5,6-16,2\end{array}$ & $\begin{array}{c}26,8 \\
20,8-33,8\end{array}$ \\
\hline NRW & $\begin{array}{c}36,7 \\
35,2-38,2\end{array}$ & $\begin{array}{c}24,1 \\
22,8-25,3\end{array}$ & $\begin{array}{c}26,8 \\
25,5-28,1\end{array}$ & $\begin{array}{c}10,2 \\
9,3-11,1\end{array}$ & $\begin{array}{c}16,1 \\
14,9-17,4\end{array}$ & $\begin{array}{c}31,3 \\
29,8-32,8\end{array}$ & $\begin{array}{c}8,7 \\
7,7-9,7\end{array}$ & $\begin{array}{c}31,2 \\
29,8-32,7\end{array}$ \\
\hline Hessen & $\begin{array}{c}34,5 \\
32,2-36,9\end{array}$ & $\begin{array}{c}21,3 \\
19,5-23,2\end{array}$ & $\begin{array}{c}26,7 \\
24,6-28,8\end{array}$ & $\begin{array}{c}11,7 \\
10,1-13,4\end{array}$ & $\begin{array}{c}14,4 \\
12,6-16,3\end{array}$ & $\begin{array}{c}28,6 \\
26,4-30,9\end{array}$ & $\begin{array}{c}8,6 \\
7,3-10,3\end{array}$ & $\begin{array}{c}27,3 \\
25,2-30,0\end{array}$ \\
\hline $\begin{array}{l}\text { Rheinland- } \\
\text { Pfalz }\end{array}$ & $\begin{array}{c}39,3 \\
36,2-42,3\end{array}$ & $\begin{array}{c}24,1 \\
21,6-26,8\end{array}$ & $\begin{array}{c}25,7 \\
23,2-28,2\end{array}$ & $\begin{array}{c}11,5 \\
9,7-13,5\end{array}$ & $\begin{array}{c}17,3 \\
14,8-20,0\end{array}$ & $\begin{array}{c}32,4 \\
29,4-35,5\end{array}$ & $\begin{array}{c}9,9 \\
7,9-12,3\end{array}$ & $\begin{array}{c}29,0 \\
26,4-32,0\end{array}$ \\
\hline $\begin{array}{l}\text { Baden - } \\
\text { Württemberg }\end{array}$ & $\begin{array}{c}31,0 \\
29,1-32,8\end{array}$ & $\begin{array}{c}19,8 \\
18,4-21,3\end{array}$ & $\begin{array}{c}22,3 \\
20,8-23,8\end{array}$ & $\begin{array}{c}10,0 \\
8,9-11,1\end{array}$ & $\begin{array}{c}13,2 \\
11,8-14,8\end{array}$ & $\begin{array}{c}28,1 \\
26,3-29,9\end{array}$ & $\begin{array}{c}7,2 \\
6,2-8,4\end{array}$ & $\begin{array}{c}25,7 \\
24,0-27,5\end{array}$ \\
\hline Bayern & $\begin{array}{c}33,1 \\
31,4-34,9\end{array}$ & $\begin{array}{c}21,5 \\
20,1-22,9\end{array}$ & $\begin{array}{c}23,0 \\
21,6-24,4\end{array}$ & $\begin{array}{c}11,1 \\
10,0-12,3\end{array}$ & $\begin{array}{c}14,7 \\
13,3-16,2\end{array}$ & $\begin{array}{c}28,0 \\
26,3-29,7\end{array}$ & $\begin{array}{c}8,3 \\
7,3-9,5\end{array}$ & $\begin{array}{c}28,6 \\
26,9-30,3\end{array}$ \\
\hline Saarland & $\begin{array}{c}38,8 \\
33,5-44,5\end{array}$ & $\begin{array}{c}21,0 \\
17,1-25,5\end{array}$ & $\begin{array}{c}24,6 \\
19,9-29,9\end{array}$ & $\begin{array}{c}14,5 \\
10,8-19,1\end{array}$ & $\begin{array}{c}14,7 \\
10,9-19,5\end{array}$ & $\begin{array}{c}30,9 \\
26,0-36,3\end{array}$ & $\begin{array}{c}9,6 \\
6,6-13,7\end{array}$ & $\begin{array}{c}34,8 \\
29,7-40,3\end{array}$ \\
\hline Berlin & $\begin{array}{c}36,4 \\
33,4-39,4\end{array}$ & $\begin{array}{c}21,4 \\
19,2-23,8\end{array}$ & $\begin{array}{c}30,9 \\
28,1-33,7\end{array}$ & $\begin{array}{c}12,8 \\
10,8-15,1\end{array}$ & $\begin{array}{c}14,5 \\
12,4-17,0\end{array}$ & $\begin{array}{c}29,7 \\
27,0-32,5\end{array}$ & $\begin{array}{c}10,3 \\
8,4-12,4\end{array}$ & $\begin{array}{c}28,2 \\
25,5-31,0\end{array}$ \\
\hline Brandenburg & $\begin{array}{c}36,0 \\
32,9-39,2\end{array}$ & $\begin{array}{c}18,3 \\
16,0-20,8\end{array}$ & $\begin{array}{c}24,2 \\
21,6-26,9\end{array}$ & $\begin{array}{c}10,6 \\
8,8-12,8\end{array}$ & $\begin{array}{c}19,1 \\
16,6-21,9\end{array}$ & $\begin{array}{c}37,2 \\
34,1-40,4\end{array}$ & $\begin{array}{c}13,2 \\
11,0-15,8\end{array}$ & $\begin{array}{c}27,3 \\
24,5-30,3\end{array}$ \\
\hline $\begin{array}{l}\text { Mecklenburg- } \\
\text { Vorpommern }\end{array}$ & $\begin{array}{c}41,2 \\
36,7-45,8\end{array}$ & $\begin{array}{c}16,6 \\
13,4-20,3\end{array}$ & $\begin{array}{c}23,2 \\
19,6-27,3\end{array}$ & $\begin{array}{c}8,3 \\
6,1--11,1\end{array}$ & $\begin{array}{c}21,3 \\
17,5-25,6\end{array}$ & $\begin{array}{c}38,9 \\
34,5-43,5\end{array}$ & $\begin{array}{c}11,6 \\
8,5-15,6\end{array}$ & $\begin{array}{c}32,0 \\
27,8-36,6\end{array}$ \\
\hline Sachsen & $\begin{array}{c}39,4 \\
36,8-44,1\end{array}$ & $\begin{array}{c}23,5 \\
21,4--25,7\end{array}$ & $\begin{array}{c}20,0 \\
18,1-22,2\end{array}$ & $\begin{array}{c}8,3 \\
6,8-10,0\end{array}$ & $\begin{array}{c}17,0 \\
15,0-19,2\end{array}$ & $\begin{array}{c}39,4 \\
36,9-42,0\end{array}$ & $\begin{array}{c}12,7 \\
10,9-14,7\end{array}$ & $\begin{array}{c}26,9 \\
24,6-29,3\end{array}$ \\
\hline $\begin{array}{l}\text { Sachsen- } \\
\text { Anhalt }\end{array}$ & $\begin{array}{c}40,8 \\
37,0-44,6\end{array}$ & $\begin{array}{c}17,1 \\
14,6-19,9\end{array}$ & $\begin{array}{c}25,1 \\
22,0-28,5\end{array}$ & $\begin{array}{c}9,5 \\
7,4-12,3\end{array}$ & $\begin{array}{c}23,2 \\
20,0-26,6\end{array}$ & $\begin{array}{c}38,4 \\
34,8-42,2\end{array}$ & $\begin{array}{c}13,6 \\
11,0-16,8\end{array}$ & $\begin{array}{c}23,2 \\
20,3-26,4\end{array}$ \\
\hline Thüringen & $\begin{array}{c}36,7 \\
33,3-40,2\end{array}$ & $\begin{array}{c}20,4 \\
17,7-23,2\end{array}$ & $\begin{array}{c}22,2 \\
19,5-25,1\end{array}$ & $\begin{array}{c}9,1 \\
7,1-11,6\end{array}$ & $\begin{array}{c}18,3 \\
15,6-21,3\end{array}$ & $\begin{array}{c}37,9 \\
34,5-41,4\end{array}$ & $\begin{array}{c}11,9 \\
9,5-14,7\end{array}$ & $\begin{array}{c}23,7 \\
20,8-26,9\end{array}$ \\
\hline $\begin{array}{l}\text { Gesamt- } \\
\text { Deutschland }\end{array}$ & $\begin{array}{c}35,3 \\
34,6-36,0\end{array}$ & $\begin{array}{c}21,7 \\
21,2-22,2\end{array}$ & $\begin{array}{c}24,9 \\
24,4-25,5\end{array}$ & $\begin{array}{c}10,4 \\
10,0-10,8\end{array}$ & $\begin{array}{c}15,7 \\
15,2-16,2\end{array}$ & $\begin{array}{c}31,1 \\
30,5-31,8\end{array}$ & $\begin{array}{c}9,2 \\
8,7-9,6\end{array}$ & $\begin{array}{c}28,2 \\
27,6-28,9\end{array}$ \\
\hline
\end{tabular}

*alle Prozentangaben sind durch Gewichtung an die Bevölkerung in Deutschland am 31.12.2011 angepasst 


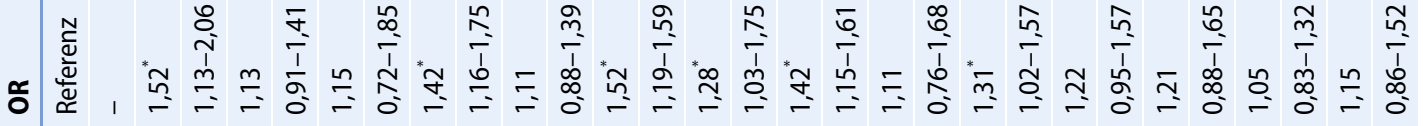

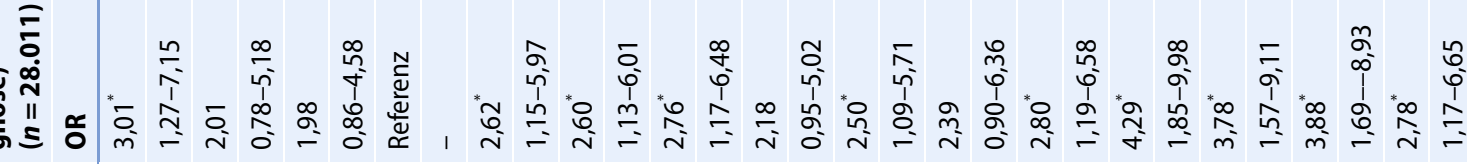

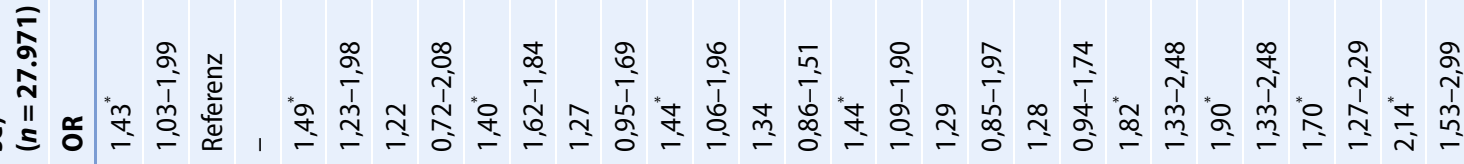

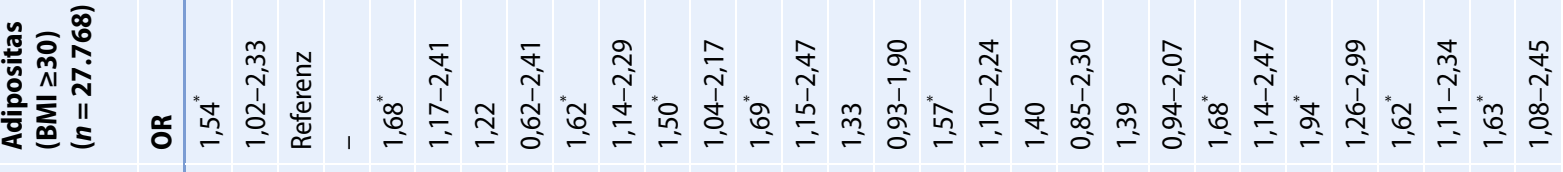
๑ั

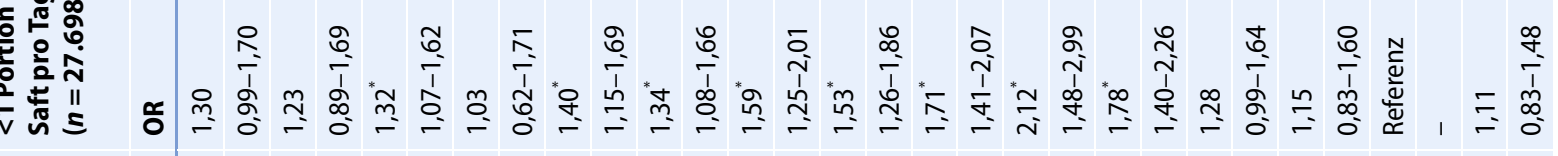

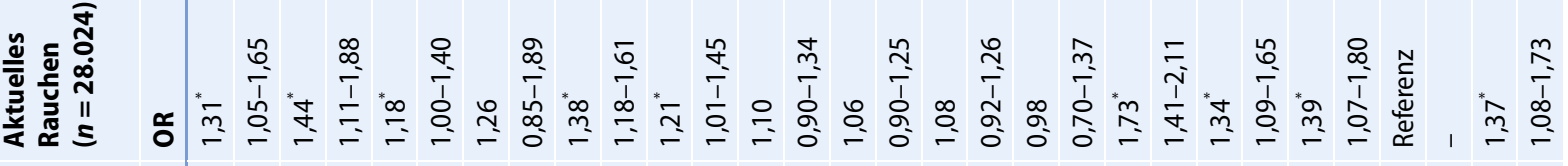

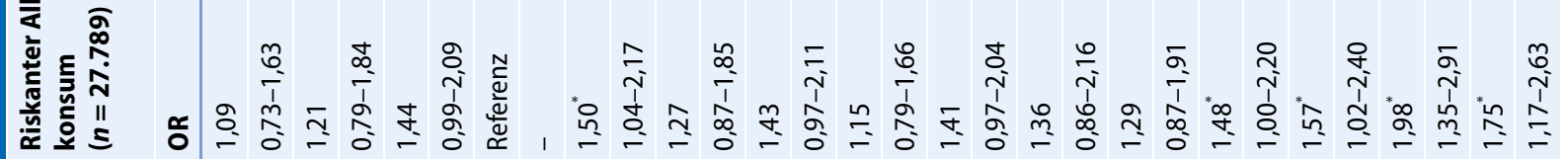




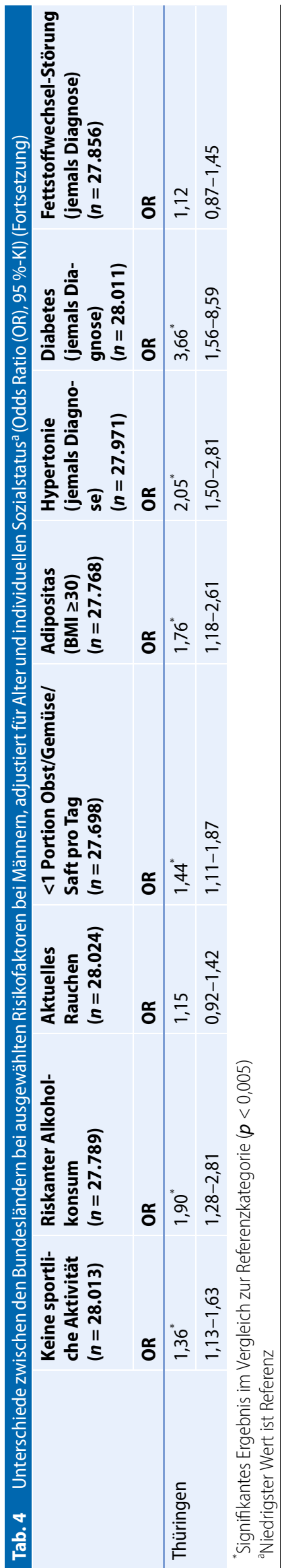

Hamburg besonders niedrig, wie bei der Adipositas und Hypertonie bei beiden Geschlechtern sowie bei riskantem Alkoholkonsum und Diabetes bei Männern. Baden-Württemberg hatte geschlechtsübergreifend den niedrigsten Anteil von sportlich inaktiven Menschen.

Insgesamt hatten Männer im Bundesdurchschnitt deutlich höhere Prävalenzen eines riskanten Alkoholkonsums (32,8\% versus $21,7 \%$ ), eines geringen Obst- und Gemüseverzehr (20,6\% versus 10,4\%) sowie des aktuellen Rauchens (32,6\% versus $24,9 \%$ ) als Frauen.

Bei einer Betrachtung der Anzahl von Risikofaktoren (• Abb. 1 und 2) lag der Anteil der Personen ohne einen einzigen Risikofaktor bei den Männern im Bundesdurchschnitt bei 13,3\% mit einer Spannweite zwischen 10,2\% in Sachsen-Anhalt und Thüringen und $16,2 \%$ in Hamburg. In den drei Ländern Mecklenburg-Vorpommern (43,5\%), Thüringen (42,9\%) und Sachsen-Anhalt $(42,3 \%)$ hatten mehr als zwei Fünftel der männlichen Einwohner drei oder mehr Risikofaktoren. In Bremen war es nur etwa jeder vierte Mann (24,2 \%). Im Bundesdurchschnitt lag dieser Anteil bei $36,0 \%$.

Beiden Frauen hatten zwischen 14,7 \% in Bremen und 22,2 \% in Baden-Württemberg keinen Risikofaktor. Im Mittel traf dies auf 18,4\% der weiblichen Bevölkerung zu. Auf der anderen Seite hatten in Sachsen-Anhalt (31,7\%), Mecklenburg-Vorpommern (31,6\%), Rheinland-Pfalz $(30,8 \%)$ sowie Brandenburg $(30,8 \%)$ mehr als ein Drittel der weiblichen Bevölkerung drei oder mehr Risikofaktoren. Der Bundesdurchschnitt lag hier bei $26,6 \%$.

\section{Diskussion}

Die vorliegende Studie zeigt anhand standardisiert erhobener Daten für die Allgemeinbevölkerung mit mehr als 62.000 Teilnehmern markante regionale Unterschiede in der Prävalenz wichtiger modifizierbarer kardiovaskulärer Risikofaktoren in Deutschland. Bei beiden Geschlechtern war der Anteil der Bevölkerung mit Adipositas, Hypertonie, Diabetes und sportlicher Inaktivität sowie bei den Männern zusätzlich noch mit riskantem Alkoholkonsum in den ostdeutschen Bundesländern mit Ausnahme von Berlin deutlich höher als im Westen. Insgesamt spiegelt die regionale Verteilung dieser Risikofaktoren die aktuelle, altersstandardisierte Mortalität (pro 100.000) an Herz-Kreislauf-Erkrankungen (ICD: I00-99) wider, die 2014 in den ostdeutschen Bundesländern (ohne Berlin) bei 450 und in den westdeutschen Bundesländern bei 378 lag [7]. Berlin passte mit nur 312 kardiovaskulären Sterbefällen - bei einem gleichzeitig günstigen Risikoprofil - nicht in dieses verallgemeinernde Ost-West-Schema. Im Einzelnen hatte Sachsen-Anhalt im Jahr 2014 mit Abstand die höchste (499) altersstandardisierte Mortalitätsrate (pro 100.000) für Herz-KreislaufErkrankungen, gefolgt von Thüringen (448), Mecklenburg-Vorpommern (443) und dann Sachsen (438). Auch bei der Lebenszeitprävalenz von bedeutsamen kardiovaskulären Erkrankungen lag Sachsen-Anhalt an der Spitze [8] der Bundesländer. Diese Zahlen stimmen mit unseren Ergebnissen überein, die Sachsen-Anhalt als einziges Bundesland zeigten, das bei zwei Risikofaktoren sowohl bei Männern (sportliche Inaktivität und Hypertonie) als auch Frauen (Adipositas und Diabetes) die höchsten Prävalenzen aufwies.

Interessanterweise hatte RheinlandPfalz in der aktuellen Analyse den dritthöchsten Anteil von Frauen mit drei oder mehr Risikofaktoren im Bundesländervergleich. Dieses Ergebnis steht im Einklang mit der zweithöchsten Lebenszeitprävalenz von kardiovaskulären Erkrankungen in der weiblichen Bevölkerung in diesem Bundesland, die in einer anderen Studie mit GEDA-Daten beobachtet wurde [8].

Die insgesamt niedrigen Prävalenzen von Risikofaktoren von Männern in Bremen und Hamburg und Frauen in Baden-Württemberg passen ebenfalls in das Bild von relativ niedrigen Morbiditätsund Mortalitätsraten für Herz-KreislaufErkrankungen in diesen Bundesländern $[4,8]$. In Bremen hingegen waren die Ergebnisse bei den Frauen widersprüchlich: Während hier bundesweit die niedrigsten Prävalenzen für Adipositas und Hypertonie gefunden wurden, war die Lebens- 


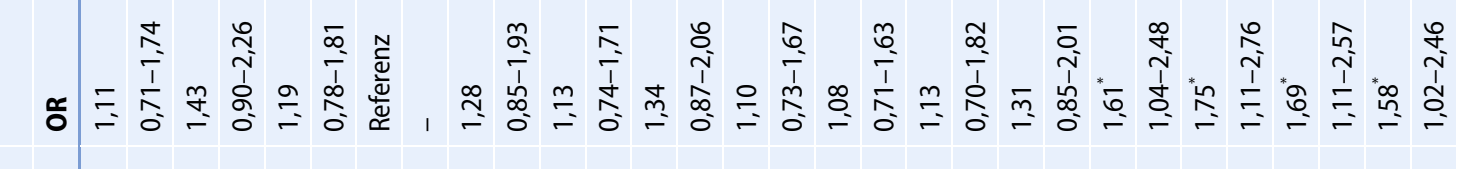

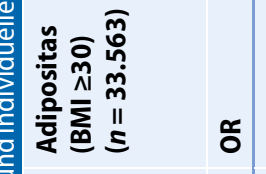

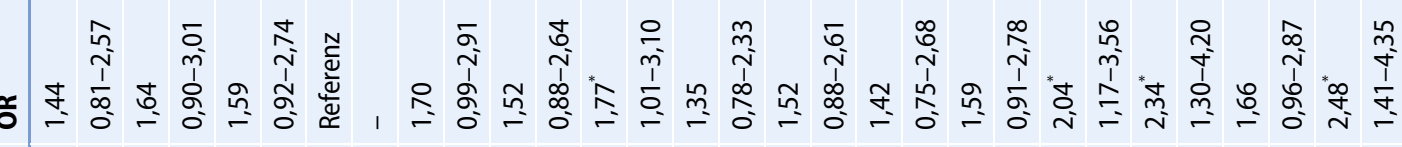




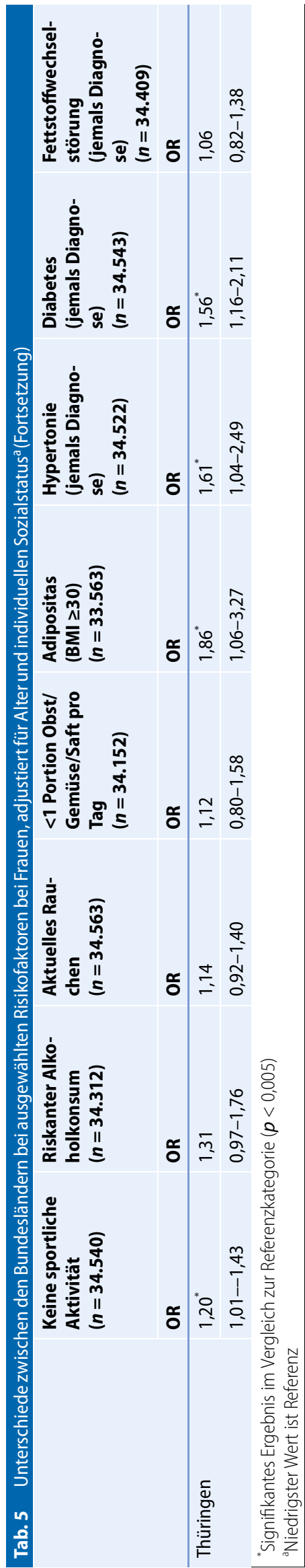

zeitprävalenz von bedeutsamen kardiovaskulären Erkrankungen vergleichsweise hoch [8].

Auch bei einem Vergleich mit anderen Datenquellen zeigen sich nach wie vor deutliche Ost-West-Unterschiede bei der Häufigkeit der diagnostizierten krankheitsnahen Risikofaktoren. In der Studie zur Gesundheit Erwachsener in Deutschland (DEGS1) war die Prävalenz der Hypertonie in Sachsen, Sachsen-Anhalt und Thüringen im regionalen Vergleich am höchsten [18]. In Hausarztpraxen (GEMCAS-Studie) wurden übereinstimmend mit unseren Ergebnissen der größte Anteil von adipösen Patienten in SachsenAnhalt (28,3\%) und der geringste in Bremen (19,8 \%) beobachtet [19]. Die Prävalenz von Diabetes war übereinstimmend in Hausarztpraxen [20], bei Mitgliedern der AOK [21] und in den nationalen Bevölkerungssurveys - Bundesgesundheitssurvey 1998 (BGS89) und DEGS1 [22] - tendenziell höher in den ostdeutschen Bundesländern.

$\mathrm{Zu}$ den verhaltensbezogenen Risikofaktoren gibt es mit Ausnahme des Tabakkonsums kaum regionale Vergleichsstudien in Deutschland. Eine gemeinsame Auswertung des BGS98 und von vier regionalen Studien zeigte ebenfalls einen höheren Anteil von Rauchern in großstädtischen Gebieten, allerdings nur bei den Frauen [23]. Im Tabakatlas [24] und in der GEMCAS-Studie war die Prävalenz von Rauchern bei Männern und Frauen in Berlin am höchsten. Sachsen hatte unter den Hausarztpatienten wie in GEDA einen vergleichsweise niedrigen Anteil von Rauchern [11]. Übereinstimmend mit den GEDA-Ergebnissen konsumierten gemäß der Nationalen Verzehrsstudie II [25] Menschen in den ostdeutschen Bundesländern tendenziell mehr Obst- und Gemüse, während vor allem der Gemüseverzehr im Saarland und in Rheinland-Pfalz geringer war.

Frauen hatten in der Regel eine niedrigere Anzahl von kardiovaskulären Risikofaktoren als Männer. Vor allem ein riskanter Alkoholkonsum, ein geringer Obst- und Gemüseverzehr und Rauchen war signifikant häufiger in der männlichen Bevölkerung, und auch der Anteil von Männern mit drei oder mehr Risikofaktoren war mit 36,0\% deutlich höher im Vergleich zu 26,6\% bei den Frauen. Diese geschlechtsspezifischen Unterschiede wurden auch in anderen Studien bestätigt [26] und passen zu den Ergebnissen einer höheren Lebenszeitprävalenz von Herz-Kreislauf-Erkrankungen bei Männern (13,3\% versus 10,7\%) [8]. Insgesamt erscheint die Prävalenz von Risikofaktoren bei Männern noch deutlicher einem Ost-West-Muster zu folgen als bei Frauen.

\section{Limitationen und Stärken}

Selbstangaben können zu einer Unterschätzung der tatsächlichen Prävalenz führen, vor allem für Alkoholkonsum [27, 28] und Adipositas [29]. Bei selbstberichteten Diagnosen werden zudem keine bislang unerkannten Erkrankungen erfasst. Hinzu kommt, dass ältere und kränkere Personen, die z.B. in Pflegeeinrichtungen leben, in der Stichprobe unterrepräsentiert sind. Dieser Selektionsbias kann eine Unterschätzung insbesondere der altersabhängigen Risikofaktoren verstärken. Auch unterschiedliche Versorgungsstrukturen in den einzelnen Bundesländern können einen Einfluss auf die Prävalenz haben. Eine überdurchschnittlich hohe Inanspruchnahme von Hausärzten, z. B. in den Stadtstaaten, könnte zu einer besseren Kontrolle der Blutwerte führen und somit die Häufigkeit des bekannten Diabetes erhöhen. Des Weiteren handelt es sich bei den Prävalenzen um Mittelwerte, die nichts über die Variationen innerhalb der einzelnen Länder aussagen. Mit Vorsicht sollte zudem die Gefahr eines ökologischen Bias bei einem Vergleich zwischen kardiovaskulären Risikofaktoren und Erkrankungen beziehungsweise Mortalität bedacht werden.

Wesentliche Stärken dieser Analyse sind das hohe Maß an Repräsentativität der Stichprobe für die Allgemeinbevölkerung in Deutschland, die Stichprobengröße mit mehr als 62.000 Teilnehmern und die Tatsache, dass die Daten zu den acht Risikofaktoren und zu den soziodemografischen Faktoren aus der gleichen hochstandardisierten Erhebung stammen. Mit den GEDA-Daten wurden in der Vergangenheit bereits regionale Vergleiche für verschiedene Risikofak- 
toren durchgeführt, allerdings mit dem Unterschied, dass die einzelnen GEDASurveys nicht gepoolt waren und damit Vergleiche im Wesentlichen auf großräumige Gebiete beschränkt waren [15]. Mit der Zusammenfassung der drei GEDASurveys konnten die Anzahl der Teilnehmer und damit die statistische Teststärke so weit erhöht werden, dass auch Vergleiche zwischen den 16 Ländern möglich waren.

\section{Fazit}

In Deutschland gibt es nach wie vor OstWest-Unterschiede mit einer höheren Prävalenz kardiovaskulärer Risikofaktoren in den ostdeutschen Ländern mit Ausnahme von Berlin. Diese Verteilung stimmt weitgehend mit den Bundeslandunterschieden bei der Prävalenz und Mortalität von Herz-Kreislauf-Erkrankungen überein $[7,8,11]$ und bleibt auch nach Adjustierung für Alter und individuellem Sozialstatus bestehen. Insgesamt gibt es aber, ungeachtet der bestehenden regionalen Disparitäten, kein Bundesland, für das nicht ein erheblicher Bedarf hinsichtlich der Prävention kardiovaskulärer Risikofaktoren bei Männern und Frauen zu konstatieren wäre. Dies gilt insbesondere, da die Mehrzahl der betrachteten Risikofaktoren durch den Lebensstil maßgeblich beeinflusst sind [26].

Daneben machen die ausgeprägten sozialen Ungleichheiten in der Betroffenheit von kardiovaskulären Risikofaktoren den Bedarf an verhältnispräventiven und zielgruppenspezifischen Maßnahmen zum Abbau gesundheitlicher Chancenungleichheiten deutlich. Auch haben soziale und gesellschaftliche Faktoren wie z. B. Arbeitslosigkeit oder auch Lebens- und Wohnverhältnisse einen weitgehenden Einfluss auf die gesundheitliche Lage und das Gesundheitsverhalten der Bevölkerung [30-33] und sollten zukünftig miteinbezogen werden. Zudem ist es wünschenswert, psychische Belastungen und Störungen, die ebenfalls als wesentliche Risikofaktoren für Herz-Kreislauf-Erkrankungen gelten, in weiteren Untersuchungen näher zu betrachten $[34,35]$ und regionale
Unterschiede innerhalb von Bundesländern zu berücksichtigen.

\section{Korrespondenzadresse}

\section{Dr. C. Diederichs}

Abteilung für Epidemiologie und Gesundheitsmonitoring, Robert Koch-Institut Postfach 650261, 13302 Berlin, Deutschland diederichsc@rki.de

\section{Einhaltung ethischer Richtlinien}

Interessenkonflikt. C. Diederichs, H. Neuhauser, L. Kroll, C. Lange, G. Mensink, C. Dornquast, C. Heidemann, C. Scheidt-Nave und M. Busch geben an, dass kein Interessenkonflikt besteht.

Dieser Beitrag beinhaltet keine von den Autoren durchgeführten Studien an Menschen oder Tieren.

\section{Literatur}

1. Mendis S, Puska P, Norrving B (2011) Global atlas on cardiovascular disease prevention and control. World Health Organization, Genf (in collaboration with the World Heart Federation and World Stroke Organization)

2. Robert Koch-Institut (2015) Gesundheit in Deutschland. Gesundheitsberichterstattung des Bundes. Gemeinsam getragen von RKI und Destatis. Robert Koch-Institut, Berlin

3. Mariotti S, Capocaccia R, Farchi G, Menotti A, Verdecchia A, Keys A (1986) Age, period, cohort and geographical area effects on the relationship between risk factors and coronary heart disease mortality. 15-year follow-up of the European cohorts of the Seven Countries study. J Chronic Dis 39:229-242

4. Robert Koch-Institut (2011) Sterblichkeit, Todesursachen und regionale Unterschiede. Heft 52. Gesundheitsberichterstattung des Bundes. Robert Koch-Institut, Berlin

5. Robert Koch-Institut (2014) 25 Jahre nach dem Fall der Berliner Mauer. Regionale Unterschiede in der Gesundheit. Gesundheitsberichterstattung des Bundes. Robert Koch-Institut, Berlin

6. Willich SN, LöwelH, MeyW, TrautnerC(1999)Regionale Unterschiede der Herz-Kreislauf-Mortalität in Deutschland. Dtsch Ärztebl 96:483-488

7. Gesundheitsberichterstattung des Bundes (2016) Tabelle: Sterbefälle, Sterbeziffern (je 100.000 Einwohner, altersstandardisiert) (ab 1998). Gliederungsmerkmale: Jahre, Region, Alter, Geschlecht, Nationalität, ICD-10, Art derStandardisierung.http://www.gbe-bund.de/oowa921-install/ servlet/oowa/aw92/dboowasys921.xwdevkit/ xwd init?gbe.isgbetol/xs start neu/\&p_aid=i\& p_aid $=50781635 \&$ nummer $=6 \&$ p_sprache $=D \&$ p_indsp $=99999999 \& p \_a i d=33431042$. Zugegriffen: 28.4.2016

8. Dornquast C, Kroll LE, Neuhauser HK, Reinhold T, Busch M (2016) Regionale Unterschiede in der Prävalenz kardiovaskulärer Erkrankungen in Deutschland - Ergebnisse der Studie zur "Gesundheit in Deutschland aktuell" (GEDA) 2009-2012. Dtsch Ärztebl 113(42):704-711. doi:10.3238/arztebl.2016.0704
9. World Health Organization (2009) Global health risks: mortality and burden of disease attributable to selected major risks. World Health Organization Genf

10. Schneider S, Huy C, Schuessler M, Diehl K, Schwarz S (2009) Optimising lifestyle interventions: identification of health behavior patterns by cluster analysis in a German 50+survey. Eur JPublic Health 19:271-277

11. Stang A, Stang M (2014) Kardiovaskuläre Risikofaktoren im Bundeslandvergleich. Ein Beitrag zu Erklärung der hohen Mortalität der ischämischen Herzkrankheit in Sachsen-Anhalt. Dtsch Ärztebl 111:530-536

12. Lange $C$, Jentsch F, Allen J et al (2015) Data Resource Profile: German Health Update (GEDA) the health interview survey for adults in Germany. Int JEpidemiol 44:442-450

13. Robert Koch-Institut (2011) Daten und Fakten: Ergebnisse der Studie "Gesundheit in Deutschland aktuell 2009". Beiträge zur Gesundheitsberichterstattung des Bundes. Robert Koch-Institut, Berlin

14. Robert Koch-Institut (2012) Daten und Fakten: Ergebnisse der Studie "Gesundheit in Deutschland aktuell 2010". Beiträge zur Gesundheitsberichterstattung des Bundes. Robert Koch-Institut, Berlin, Deutschland

15. Robert Koch-Institut (2014) Daten und Fakten: Ergebnisse der Studie "Gesundheit in Deutschland aktuell 2012". Beiträge zur Gesundheitsberichterstattung des Bundes. Robert Koch-Institut, Berlin, Deutschland

16. Bush K, Kivlahan DR, McDonell MB, Fihn SD, Bradley KA (1998) The AUDIT alcohol consumption questions (AUDIT-C): an effective brief screening test for problem drinking. Ambulatory Care Quality Improvement Project (ACQUIP). Alcohol use disorders identification test. Arch Intern Med 158:1789-1795

17. Lampert T, Kroll LE, Müters S, Stolzenberg H (2013) Messung des sozioökonomischen Statusin derStudie "Gesundheit in Deutschland aktuell" (GEDA). Bundesgesundheitsblatt Gesundheitsforschung Gesundheitsschutz 56:131-143

18. Diederichs C, Neuhauser H (2014) Regional variations in hypertension prevalence and management in Germany: results from the German Health Interview and Examination Survey (DEGS1). JHypertens 32:1405-1413

19. Hauner $H$, Bramlage $P$, Lösch $C$ et al (2008) Übergewicht, Adipositas und erhöhter Taillenumfang - Regionale Prävalenzunterschiede in der hausärztlichen Versorgung. Dtsch Ärztebl 105:827-833

20. Moebus S, Hanisch J, Bramlage P et al (2008) Regional differences in the prevalence of the metabolic syndrome in primary care practices in germany. Dtsch Ärztebl Int 105:207-213

21. Müller N, Heller T, Freitag MH et al (2015) Healthcare utilization of people with Typ 2 diabetes in Germany: an analysis based on health insurance data. Diabet Med 32:951-957

22. Heidemann C, Du Y, Paprott R, Haftenberger M Rathmann W, Scheidt-Nave C (2015) Temporal changes in the prevalence of diagnosed diabetes, undiagnosed diabetes and prediabetes: findings from the German Health Interview and Examination Surveys in 1997-1999 and 2008-2011. Diabet Med 33:1406-1414

23. Völzke H, Neuhauser H, Moebus S et al (2006) Rauchen: Regionale Unterschiede in Deutschland. Dtsch Ärztebl 103:2784-2790 
24. Deutsches Krebsforschungszentrum (2015) Tabakatlas Deutschland 2015. Deutsches Krebsforschungszentrum, Heidelberg

25. Max Rubner-Institut, Bundesforschungsinstitut für Ernährung und Lebensmittel (2008) Nationale Verzehrstudie - Ergebnisbericht, Teil 2. Max Rubner-Institut, Bundesforschungsinstitut für Ernährung und Lebensmittel, Karlsruhe

26. Linardakis M, Papadaki A, Smpokos E, Micheli K, Vozikaki M, Philalithis A (2015) Association of behavioral risk factors for chronic diseases with physical and mental health in European adults aged 50 years or older. Prev Chronic Dis 12(150134):2004-2005

27. Stockwell T, Zhao J, Macdonald S (2014) Who under-reports their alcohol consumption in telephone surveys and by how much? An application of the ,yesterday method' in a national Canadian substance use survey. Addiction 109:1657-1666

28. Bellis MA, Hughes K, Cook PA, Morleo M (2009) Off measure: How we underestimate the amount we drink. Alcohol Concern, London

29. Gorber SC, Tremblay M, Moher D, Gorber B (2007) A comparison of direct vs. self-report measures for assessing height, weight and body mass index: a systematic review. Obes Rev 8:307-326

30. Wamala SP, Mittleman MA, Schenck-Gustafsson K, Orth-Gomér K (1999) Potential explanations for the educational gradient in coronary heart disease: a population-based case-control study of Swedish women. Am J Public Health 89:315-321

31. Palomo L, Félix-Redondo FJ, Lozano-Mera L, PérezCastán JF, Fernández-Berges D, Buitrago F (2014) Cardiovascular risk factors, lifestyle, and social determinants. BrJGen Pract 64:e627-e633

32. Gascon M, Triguero-Mas M, Martínez D et al (2016) Residential green spaces and mortality: a systematic review. Environ Int 86:60-67

33. Newton JN, Briggs ADM, Murray C et al (2013) Changes in health in England, with analysis by English regions and areas of deprivation, 1990-2013: a systematic analysis for the Global Burden of Disease Study 2013. Lancet 386:2257-2274

34. Deutsche Herzstiftung e. V. (2015) 27. Deutscher Herzbericht 2015. Sektorenübergreifende Versorgungsanalyse zur Kardiologie und Herzchirurgie in Deutschland. Deutsche Herzstiftung e.V., Frankfurta. M.

35. Yusuf S, Hawken S, Ounpuu S et al (2004) Effect of potentially modifiable risk factors associated with myocardial infarction in 52 countries (the INTERHEART study): case-control study. Lancet 364:937-952 